\title{
APPLYING WEB SERVICES TECHNOLOGY TO IMPLEMENT DISTRIBUTED SIMULATION FOR SUPPLY CHAIN MODELING AND ANALYSIS
}

\author{
Taejong Yoo \\ Kyungdoc Kim \\ Sunyoung Song \\ Hyunbo Cho \\ Dept. of Industrial and Management Engineering \\ POSTECH
}

San 31, Hyoja, Pohang 790-784, Republic of Korea

\author{
Enver Yücesan \\ Technology and Operation Management Area \\ INSEAD \\ Boulevard de Constance 77305, Fontainebleau Cedex, \\ France
}

\begin{abstract}
The introduction of Parallel and Distributed Simulation (PADS) has added great impetus to efforts to use simulation as a strategic tool to support decision making in supply chain management. However, due to the heterogeneity and the dynamic nature of supply chains, there are many challenges that must be overcome if supply chain simulation is to play an effective role. This paper describes the application of web services technology to the domain of supply chain simulation. A supply chain simulation framework is proposed through a combination of PADS and web services technology. In the proposed framework, PADS provides the infrastructure for supply chain simulation execution while web services technology makes it possible to coordinate the supply chain simulation model. A prototype implementation with a simple supply chain simulation model demonstrates the viability of the proposed supply chain simulation framework.
\end{abstract}

\section{INTRODUCTION}

In a fiercely competitive business environment, effective supply chain management is crucial for a business to provide customers with products and/or services faster and better than its competitors. Since a supply chain consists of geographically distributed facilities and transportation links connecting these facilities, a principal aim of supply chain management is to create agile groups of independent but cooperating participants, making it possible to reduce costs and increase competitiveness. This challenge is complicated by various factors, including the wide range of products, complex processes at all stages, and elaborate interfaces with suppliers and customers.

Simulation holds great potential as a means of portraying the dynamic evolution of supply chains and of providing dynamic decision support to address challenges arising from high variability and stochastic uncertainty. Traditionally, a supply chain involves only a single enterprise with multiple facilities and distribution centers. Under this centralized scenario, building and sharing detailed simulation models is not a problem. Recently, however, the scope of supply chain management has evolved to cross enterprise boundaries. Applying simulation in designing, evaluating, and optimizing a decentralized supply chain may be difficult in cases where the participating corporations are unwilling to share their simulation models with partners (Gan, et al. 2000). Another handicap is the lack of simulation environments facilitating such collaboration: while there are 65 major commercial simulation tools, only 9 of those tools are capable of supply chain simulation (Swain 2007). Furthermore, even commercial simulation tools that have a supply chain simulation module can only support a single centralized model that encompasses multiple participants in the supply chain. In terms of simulating supply chain dynamics, newer simulation concepts such as agent-based or distributed simulation for interoperability and reusability remain topics of research that have yet to be incorporated into commercial tools. It is also desirable to realize high-fidelity supply chain simulations through timely and accurate information exchange among all participants of the supply chain. Overall, no fully established simulation package is available to guide and assist in supply chain simulation.

Parallel and Distributed Simulation (PADS) has immense potential as a tool for supply chain simulation. Although the fundamental concept of distributed simulation is quite convenient for simulating supply chains, problems remain regarding simulation model composition, the integration of different models, and communication among models. In the heterogeneous 
computing environment of supply chain participants, web services technology can be employed to seamlessly integrate and operate existing simulation models in a platform-independent manner. Web services represent a paradigm for building distributed applications from a collection of services. Each service is considered as a self-contained, self-describing, modular component. As a consequence, web services provide a natural modeling framework for depicting business ecosystems. This is further facilitated by the use of common standard conventions. For instance, the message object exchanged among all services is typically developed using the business document standards such as the United Nations electronic Trade Document (UNeDocs) for e-business environment (UN/CEFACT 2005).

The objective of the present study is to propose an interoperable and reusable supply chain simulation framework. In particular, since the proposed simulation framework is the initial stage of these research fields, this paper just concentrates the heterogeneous simulation model building using web services technologies. As a proof of concept, we introduce a supply chain simulation modeling methodology where all simulation-related components are implemented as a service component.

The remainder of the paper is organized as follows. Section 2 presents a survey of the relevant literature. Section 3 introduces the framework of web services for supply chain simulation. The prototype implementation of a simple case is described in Section 4. Finally, Section 5 summarizes current research efforts and future work.

\section{RELATED WORK}

\subsection{Supply Chain Simulation}

Modeling of supply chains is different from modeling of traditional manufacturing systems. Conventional simulations of manufacturing systems focus on the material flow through different machines and material handling systems. Parts release is usually modeled using an inter-arrival time distribution or some basic rules. These models provide value through the determination of machine utilizations, cycle times, and bottleneck analysis. By contrast, a supply chain simulation model should be built by integrating models of manufacturing and logistics systems only if the component models include sub-models of the business processes and information flows in addition to material flows (Banks, et al. 2002).

The methodology required to realize a supply chain simulation can be separated into two categories: a single model reproducing all nodes (a.k.a. local simulation) and several models running in parallel in a single coordinated environment (a.k.a. parallel and distributed simulation (Fujimoto 1999, Yücesan, et al. 2001, Page, et al. 1999)). PADS has come to be considered the most viable supply chain simulation tool. This is due to its significant advantages (Chandra and Chilov 2001, McLean and Riddick 2000, Terzi and Cavalieri 2004):

- PADS makes it possible to realize complex simulation models that cross enterprise boundaries without any need for sharing of local production system models and data.

- PADS paradigm guarantees the "encapsulation" of different local models within one overall complex simulation system so that, apart from the information exchanged, each model is self-contained.

- PADS provides a connection between supply chain participants that are geographically distributed throughout the globe, guaranteeing that each single simulation model is linked to its respective industrial site.

Recent developments in Information Technology (IT) have revived interest in PADS through the introduction of simulation languages and the building of model libraries that can be assembled and executed over the world wide web (WWW). However, implementation of PADS over the WWW requires a platform for synchronization and data sharing, as well as the development of interfaces to allow for integration of the models in a shared architecture and for reliability of data transfer. Consequently, researchers seeking to achieve such a PADS implementation are faced with the inescapable challenge to achieve interoperability among heterogeneous computing platforms. The majority of work done in supply chain simulation is the area of model distribution. The execution of simulations on distributed hosts as a coupled model requires both coordination and facilitating infrastructure. Although there are some approaches for PADS such as HLA or GRIDS-SCF, the interoperability problem still remain as a major issue (Taylor and Sudra 2002, Taylor, et al. 2002).

\subsection{Web Services Technology}

Web services, which implement the concept of integrating software applications as a service, represent universally accessible software components deployed on the WWW. Such a software component is described by an interface listing the collection of operations that can be performed on it (Chandrasekaran, et al. 2002). Web services allows applications to communicate with other applications using open standards (Tsalgatidou and Pilioura 2002), even if they are running on different operating systems or are written in different languages. Due to these characteristics, there exist significant synergies between web services and distributed simulation (Kilgore 2002). The potential contributions of web services to simulation include: 
- Faster execution times of a simulation experiment through distribution of runs and alternatives to banks of available processors.

- Geographic distribution of the simulation to allow more convenient collaboration.

- Integration of simulations on different hardware devices and operating systems, particularly in training applications.

- Integration of actual systems and simulated systems for test and evaluation or control.

Service-oriented architecture (SOA) has emerged, as a new software development paradigm in recent years, in which major activities in application building are integrating existing services to deliver required functionalities. New simulation environments must provide facilities to simulate such activities of SOA based applications. Pioneering work in this direction has been done in Cosim-grid and Aurora (Li, et al. 2005, Park and Fujimoto 2006)

\section{APPLYING WEB SERVICES FOR SUPPLY CHAIN SIMULATION}

In the present work, an overall framework is proposed to model supply chain simulation. The proposed framework is a complex simulation environment that interprets various inputs and returns the performance results for each supply chain participant.

The basic concept of the proposed framework is shown in Figure 1. Each participant in a supply chain provides a simulation service that represents its internal simulation model, where the simulation service is deployed using web services technology. According to the simulation service implementation details, the supply chain simulation modeler can instantiate the supply chain simulation model by invoking the simulation service of each participant.

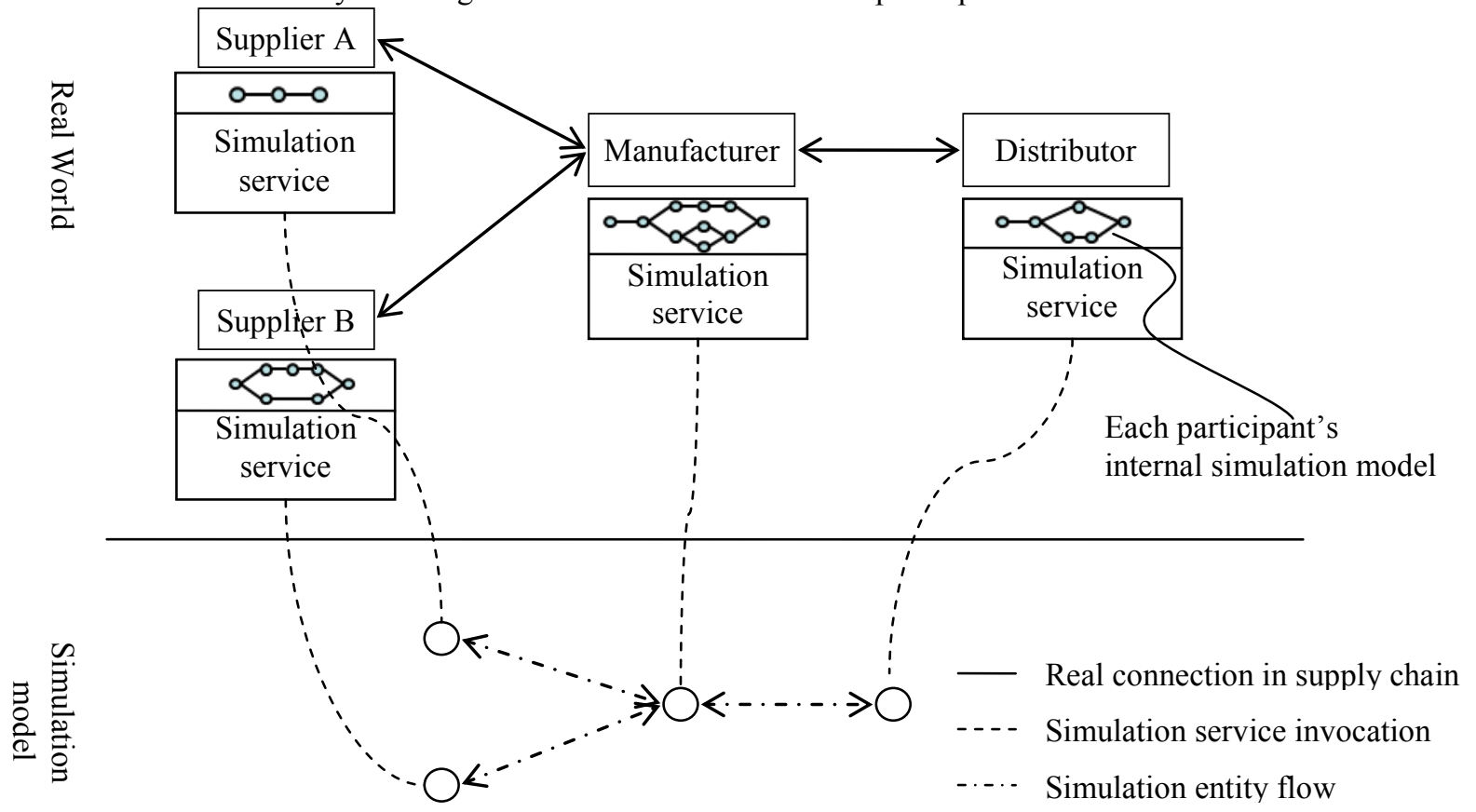

Figure 1: Supply chain simulation framework applying web services

\subsection{Simulation Service}

In the WWW environment, the remote servers developed by supply chain participants must be coordinated. For reusable and interoperable supply chain simulations, the Simulation Service can be defined with web services as a means to access remote execution engines, as shown in Figure 2. A core simulation engine, which should be the simulation model of each supply chain participant, is built on top of SOAP-based message processing that, in turn, is based on web services technology. The SOAP message from the service requestor is translated into the input data type of the core simulation engine through the message transaction module. Through the input interface, the core simulation engine receives the input data and performs the requested operations. Through the output interface, the SOAP-based message processing receives the output data and sends the SOAP message, which is translated by the message transaction. This approach enables both the use of an existing simulation system as a core simulation engine and communication of the simulation service with other services without any additional effort. Therefore, it naturally supports communication among heterogeneous simulation engines. 
All simulation services exchange the SOAP message that brings the payload that contains the simulation entity. The simulation entity should represent the product flow, the information flow, and the cash flow. In the proposed simulation service, the schema of payloads is developed under the standard and the recommendation of UNeDocs. Since UNeDocs standard supports the generation and exchange of standardized business documents, the proposed simulation framework can be applied to the standard based e-business environment without the huge implementation problem. Table 1 shows an illustrative schema definition for the payload of the simulation service. It contains the information such as product quantity, product price, production duration, delivery duration, delivery cost that should be defined according to UNeDocs data model.

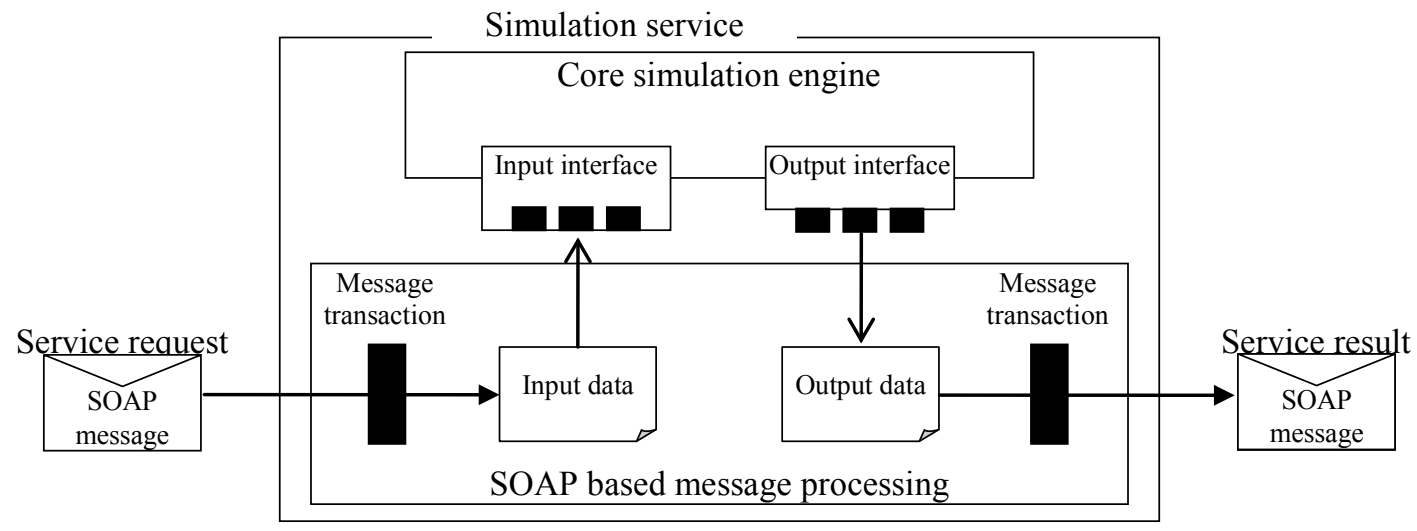

Figure 2: Simulation service architecture

Table 1: Illustrative schema definition of payload using UNeDocs data model

\begin{tabular}{|c|c|c|c|}
\hline $\begin{array}{l}\text { The information in } \\
\text { the payload }\end{array}$ & $\begin{array}{l}\text { Unique UN } \\
\text { Assigned ID }\end{array}$ & Dictionary Entry Name & Definition Mandatory \\
\hline Product quantity & UN01004326 & $\begin{array}{l}\text { Supply Chain_Supply Plan. Actual. } \\
\text { Quantity }\end{array}$ & $\begin{array}{l}\text { The actual quantity in this supply chain } \\
\text { supply plan }\end{array}$ \\
\hline Product price & UN01004860 & $\begin{array}{l}\text { Supply Chain_Trade Agreement. } \\
\text { Agreed Price Product. Trade Price }\end{array}$ & $\begin{array}{l}\text { The agreed price for the product in this } \\
\text { supply chain trade agreement }\end{array}$ \\
\hline Production duration & UN01001271 & Specified_Period. Duration. Measure & A measure of the length of time for this \\
\hline Delivery duration & & & $\begin{array}{l}\text { specified time period such as hours, days, } \\
\text { weeks, months, years }\end{array}$ \\
\hline Delivery cost & UN1004375 & $\begin{array}{l}\text { Supply Chain_Trade Delivery. Billed. } \\
\text { Quantity }\end{array}$ & $\begin{array}{l}\text { The quantity billed for this supply chain } \\
\text { trade delivery }\end{array}$ \\
\hline
\end{tabular}

\subsection{Supply Chain Simulation Modeling}

The main focus of supply chain simulation modeling is the methodology to construct and execute a supply chain simulation model. Because the proposed simulation framework is based on web services technology and is aimed at supporting business collaboration, the current business collaboration technology can be adopted as a modeling and execution tool. Business Process Modeling Notation (BPMN) (OMG 2006) can be used to construct the supply chain simulation model, and Business Process Execution Language (BPEL) (OASIS 2007). can be adopted to execute the simulation model.

The user of the proposed simulation framework (i.e. the decision maker for supply chain configuration or the simulation service requester) has three steps as shown in Figure 3.

Create the abstract supply chain simulation model by BPMN

A simple example of BPMN supply chain model is depicted in Figure 4. There are two suppliers, one manufacturer, one distributor, and two retailers. Since the simulation model is constructed by the combination of simulation units (e.g. supplier, manufacturer, distributor, and retailer), the supply chain simulation model is also constructed by the combination of simulation service. In the example supply chain simulation model, the current problem is to find the best collaborative partner for the Supplier A spot and Distributor.

Generate the BPEL document 
The user generates BPEL models as alternative simulation models with all implementation details of the simulation service from candidate supply chain participants. In this step, all candidate alternatives will be generated. In the above simple case, all supply chain participants are fixed except for Supplier A and Distributor. If the number of the candidate supplier is three and the number of the candidate distributor is four, the number of all candidate supply chain simulation model can be twelve and twelve different BPEL documents will be generated for simulation. In particular, for time management, all BPEL documents should invoke the time management service that is the organizer to make an earliest event through whole simulation model.

Execute the BPEL document

Once the BPEL document is generated, it is deployed to the BPEL execution engine such as Oracle BPEL Process Manager. It is a scalable and easy to deploy infrastructure for modeling, connecting, deploying and managing BPEL processes. Since the BPEL execution engines have been developed by several software vendors, the decision maker can execute BPEL document without any trouble. On the other hand, the entire BPEL process is also deployed as a Web Services. It has its own WSDL and waits for an incoming SOAP message to start.

1. Create the abstract supply chain simulation model by BPMN

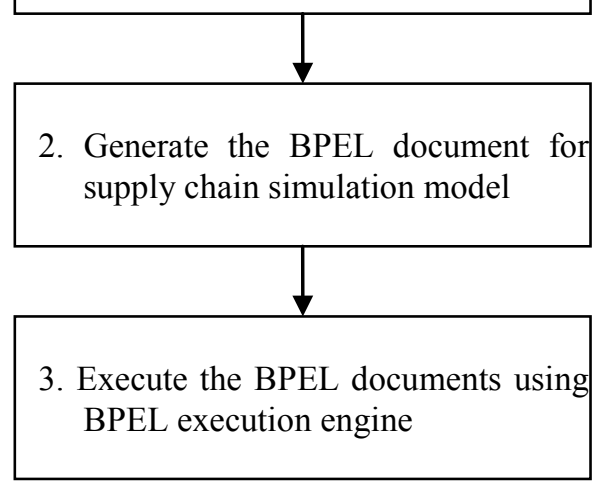

Figure 3: Simulation modeling and execution steps

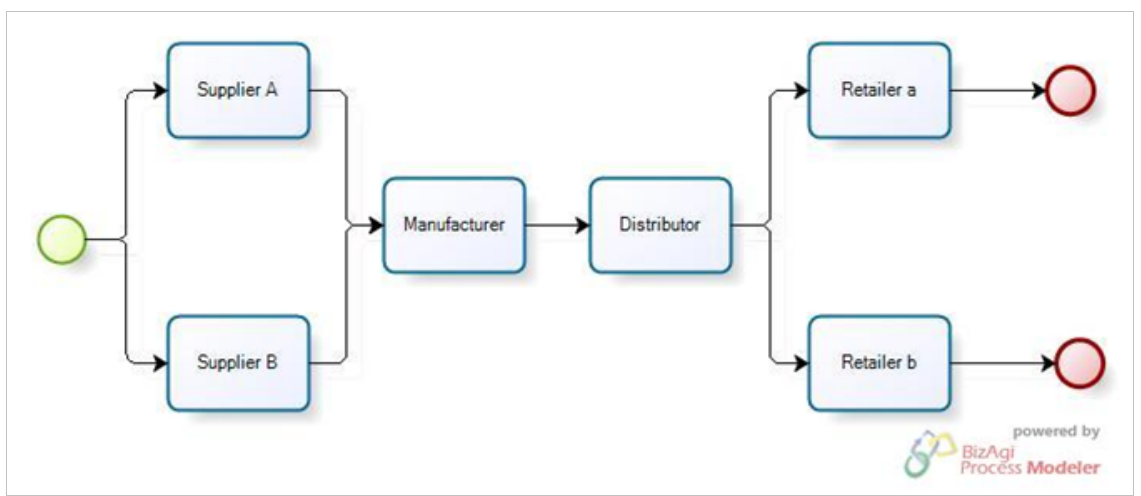

Figure 4: The example of the abstract supply chain model by BPMN

\subsection{HLA based Time Management Service}

The orchestration architecture in BPEL is same with the RTI architecture of HLA as shown in Figure 5. In RTI, all simulation federates and RTI exchange the time status and rearrange the event according to the time sequence. In SOS-SC, as RTI in HLA, RTI service is developed. When every message exchange event occurs between BPEL execution engine and simulation service, RTI service checks the time stamps from all simulation services and make a priority according to the time ascending order. Then, BPEL execution engine sends the message to simulation server that has the earliest time stamp.

RTI service can be developed as the service or the add-in programs in BPEL execution engine. Keep the time stamp of simulation entity from all simulation service and find the priority of next processes. The mechanism of RTI service is simply 
formalized as follows: After getting $i$ by equation (1), BPEL execution engine decides the next simulation service will be invoked.

$$
i=\arg \min \left(t_{i j}\right), \text { for all } i
$$

Where $t_{i j}$ is the time stamp of $j^{t h}$ simulation entity in $i^{\text {th }}$ simulation server

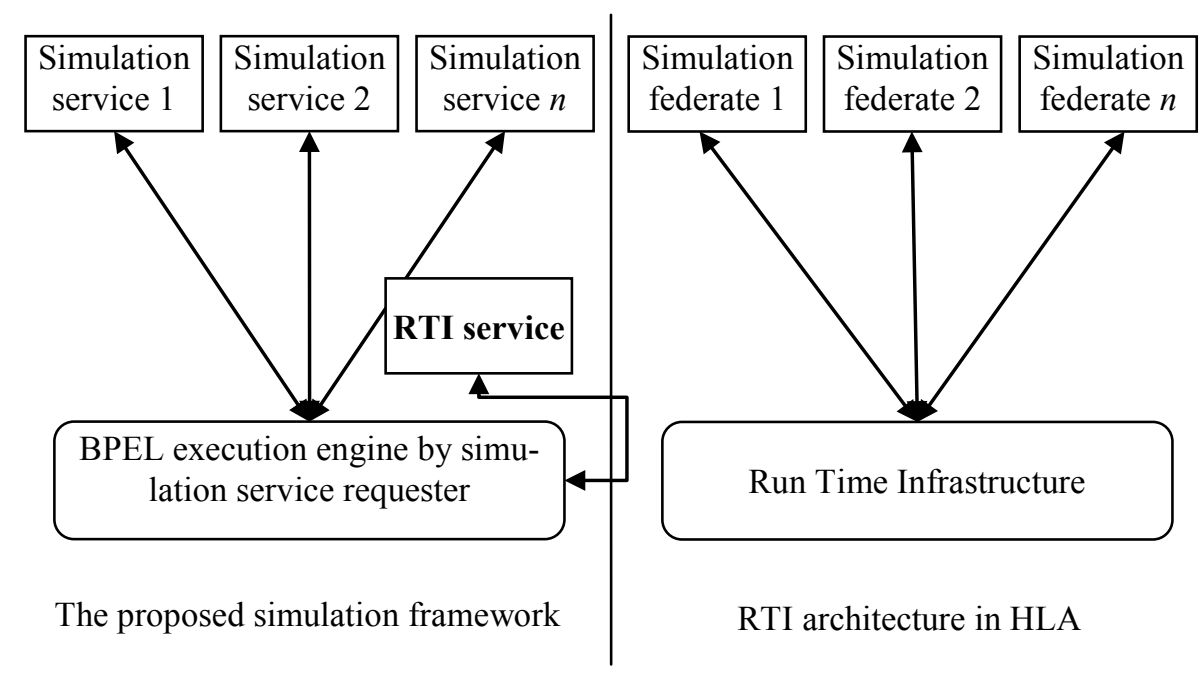

Figure 5: The architecture comparison between proposed simulation framework and RTI

\section{EXPERIMENT AND IMPLECATIONS}

The objective of experiment is to demonstrate the principal application of the proposed simulation framework for the supply chain simulation. Assumed that the simulation services are provided by virtual companies and the supply chain simulation model is generated by using the proposed framework. Oracle BPEL Process Manager is adopted as a simulation modeling and execution tool. Oracle BPEL Process Manager is a BPEL engine that is a member of the Oracle Fusion Middleware family of products. It provides a framework for designing, deploying, monitoring, and administering processes based on BPEL standards (Oracle 2005). It includes the Oracle Business Process Analysis Suite that supports graphical modeling based on BPMN. Through the Oracle BPEL tool, a BPMN based model is translated into BPEL and executes the modeled process.

\subsection{Experimental Model Assumption}

As an experimental model, a simple supply chain network is developed. Figure 6 shows the abstract supply chain model designed by Oracle BPEL Process Manager. To create a virtual supply chain situation, 3 computing machines are adopted. The simulation model of the manufacturer and distributor is located on the same computing machine $(2.0 \mathrm{GHz} \mathrm{CPU}$ and 512 SDRAM). The simulation models of the supplier of part type A and of the supplier of part type B are implemented as a simulation service by 2 computing machines each with $600 \mathrm{MHz}$ CPU and 512 SDRAM. The simulation services of the two suppliers and the factory are deployed using web services technology.

From the abstract simulation model shown in Figure 7, BPEL simulation models can be generated with invocation of simulation services. In the prototype implementation, Invoke_s11 and Invoke_s21 will invoke the simulation services of the candidate suppliers. Figure 5 depicts the partnerLink code in BPEL when the simulation services of s11 and s21 are invoked. There are 3 invocations of simulation services, from the part A supplier, the part B supplier and the factory, respectively. In the experimental model, there are 3 candidate suppliers for each part type, denoted SA1, SA2, and SA3 for part A suppliers, and SB1, SB2, and SB3 for part B suppliers. Since there are a total of 9 alternatives, 9 different BPEL codes are generated and executed.

Under conditions of a fixed manufacturer and distributor, the purpose of the simulation is to identify which suppliers are better. That is, the objective of the simulation for the manufacturer is to find the best combination of suppliers that maximizes 
manufacturer profit. Note that the production plan of the manufacturer is to produce 12,000 final product units per 30 days. The detailed simulation model assumptions are as follows:

(a) Supply chain performance criterion: profit in a given time (profit $=$ sales - total cost).

(b) The production policy of all participants is make-to-stock.

(c) Sales are calculated based on the number of product units produced on time (the price of each product unit is $\$ 150)$.

(d) Total cost is calculated based on the contract costs with the suppliers, inventory costs, and transportation costs.

(e) The detailed contract costs are summarized in Table 2.

(f) The inventory costs for parts $\mathrm{A}$ and $\mathrm{B}$ are $\$ 0.1$ and $\$ 0.5$ per day, respectively.

(g) The final product is assembled using 2 parts from part A supplier and 1 part from part B supplier.

(h) The assembly capability of the manufacturer is 400 product units per day.

(i) Each supplier has different part costs and production policies.

(j) The transportation cost depends on the location of the supplier and the weight of the parts.

(k) Supply chain flow breakdowns are not considered.

(l) Communication breakdowns from simulation service are not considered.

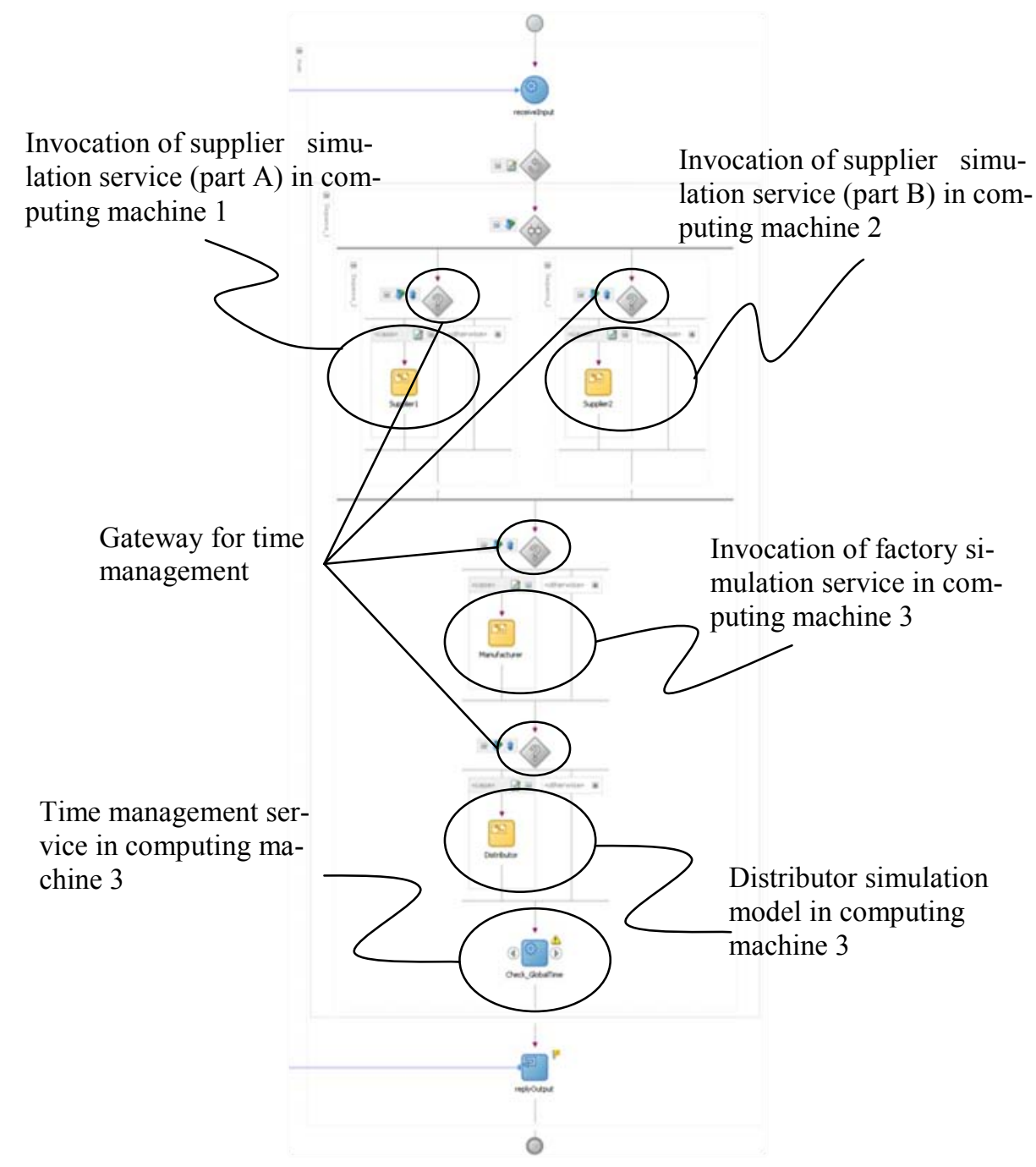

Figure 6: Abstract supply chain model designed by Oracle BPEL Process Manager

In the abstract supply chain model designed by Oracle BPEL Process Manager, simulation services from all supply chain participants are invoked within gateway. In the gateway, the current simulation time of supply chain simulation model and 
the local simulation time of each simulation service are compared and simulation service is performed when the local simulation time is later than the current simulation time. Then, time management service decides the current simulation time.

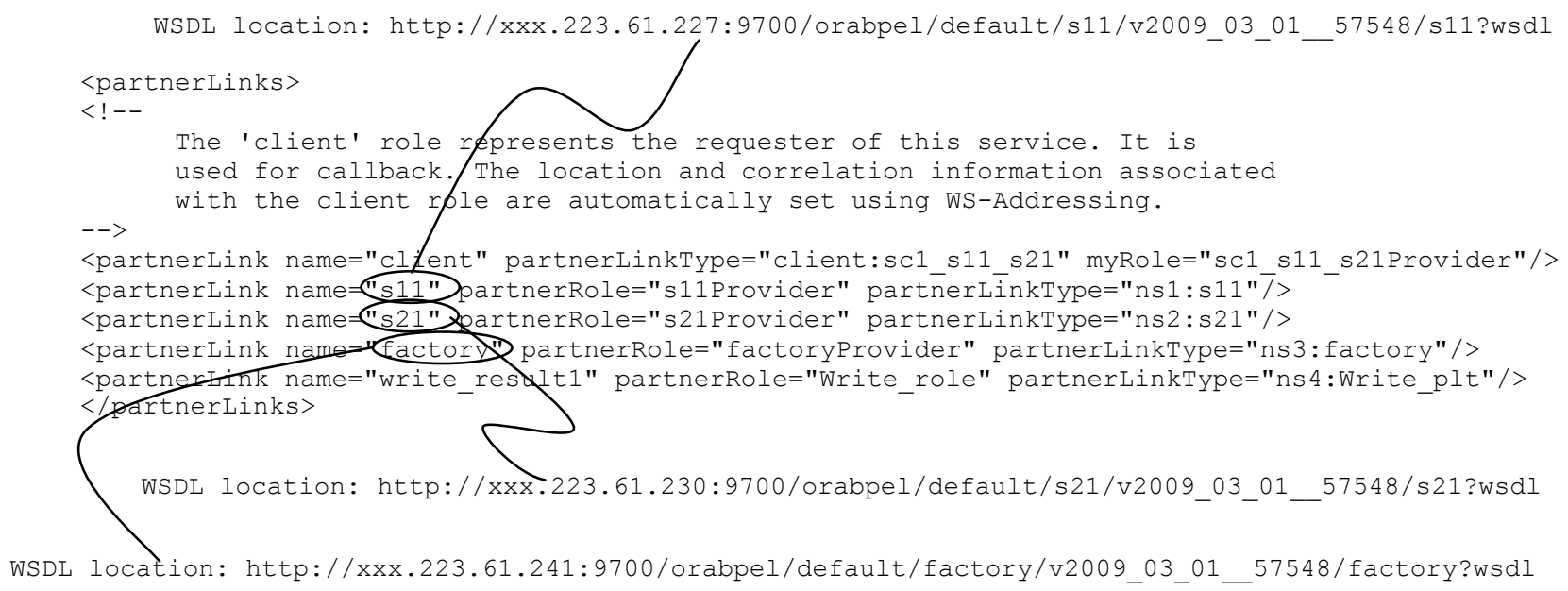

Figure 7: BPEL and WSDL of prototype implementation

Table 2: Contract condition of suppliers

\begin{tabular}{llllll}
\hline Supplier & $\begin{array}{l}\text { Total supply } \\
\text { per month }\end{array}$ & $\begin{array}{l}\text { Cost }(\$) \\
\text { per month }\end{array}$ & Batch size & $\begin{array}{l}\text { Delivery frequency } \\
\text { per month }\end{array}$ & $\begin{array}{l}\text { Transportation cost }(\$) \\
\text { per one delivery }\end{array}$ \\
\hline SA1 & 24000 & 100000 & 800 & 30 & 550 \\
SA2 & 24000 & 110000 & 1,600 & 15 & 1,050 \\
SA3 & 27000 & 115000 & 900 & 30 & 600 \\
SB1 & 12000 & 200000 & 400 & 30 & 1,000 \\
SB2 & 12000 & 200000 & 800 & 15 & 1,950 \\
SB3 & 15000 & 210000 & 500 & 30 & 1,100 \\
\hline
\end{tabular}

\subsection{Result and Implication}

Intuitively, the combination of SA1 and SB1 looks the best due to the minimum cost and the similar batch size with the manufacturer. However, simulation of the 9 combinations of candidate suppliers reveals that this combination of suppliers is not optimal over all timescales. Table 3 lists the total cost, total sales, and the resulting profit over 4 periods: 3, 6, 12, and 24 months. All simulation results are derived from 100 simulation replications. During all of the periods, the combination of SA1 and SB2 has the lowest cost and the combination of SA3 and SB3 shows the biggest sales. Nonetheless, in line with the intuitive interpretation, the combination of SA1 and SB1 provides the highest profit over 3 and 6 months. Over 12 and 24 months, however, the combination of SA1 and SB2 provides the highest profit. Thus, from the viewpoint of product life cycle, if the product has a short life cycle (up to 1 year), the combination of SA1 and SB1 should be selected. If, however, the supply chain will produce the product for more than one year, the manufacturer should use SA1 and SB2. 
Yoo, Kim, Song, Cho and Yücesan

Table 3: Simulation result for all combinations of suppliers in given period

\begin{tabular}{|c|c|c|c|c|}
\hline Period & Supplier combination & Total sales & Total cost & $\begin{array}{l}\text { Profit } \\
=(\text { sales }- \text { cost })\end{array}$ \\
\hline \multirow[t]{9}{*}{3 months } & SA1 \& SB1 & $\$ 5,320,500$ & $\$ 1,044,892$ & $\$ 4,275,608$ \\
\hline & SA1 \& SB2 & $\$ 5,284,560$ & $\$ 1, \mathbf{0 3 7 , 5 3 3}$ & $\$ 4,247,027$ \\
\hline & SA1 \& SB3 & $\$ 5,335,800$ & $\$ 1,283,591$ & $\$ 4,052,209$ \\
\hline & SA2 \& SB1 & $\$ 5,287,440$ & $\$ 1,073,401$ & $\$ 4,214,039$ \\
\hline & $\mathrm{SA} 2 \& \mathrm{SB} 2$ & $\$ 5,258,760$ & $\$ 1,062,888$ & $\$ 4,195,872$ \\
\hline & SA2 \& SB3 & $\$ 5,301,240$ & $\$ 1,313,804$ & $\$ 3,987,436$ \\
\hline & $\mathrm{SA} 3 \& \mathrm{SB} 1$ & $\$ 5,335,260$ & $\$ 1,133,153$ & $\$ 4,202,107$ \\
\hline & SA3 \& SB2 & $\$ 5,289,960$ & $\$ 1,124,876$ & $\$ 4,165,084$ \\
\hline & $\mathrm{SA} 3 \& \mathrm{SB} 3$ & $\$ 5,359,620$ & $\$ 1,370,988$ & $\$ 3,988,632$ \\
\hline \multirow[t]{9}{*}{6 months } & SA1 \& SB1 & $\$ 10,719,300$ & $\$ 2,090,637$ & $\$ 8,628,663$ \\
\hline & SA1 \& SB2 & $\$ 10,686,720$ & $\$ 2,074,189$ & $\$ 8,612,531$ \\
\hline & SA1 \& SB3 & $\$ 10,734,780$ & $\$ 2,975,071$ & $\$ 7,759,709$ \\
\hline & SA2 \& SB1 & $\$ 10,689,540$ & $\$ 2,148,374$ & $\$ 8,541,166$ \\
\hline & $\mathrm{SA} 2 \& \mathrm{SB} 2$ & $\$ 10,664,160$ & $\$ 2,128,556$ & $\$ 8,535,604$ \\
\hline & SA2 \& SB3 & $\$ 10,696,320$ & $\$ 3,031,506$ & $\$ 7,664,814$ \\
\hline & SA3 \& SB1 & $\$ 10,734,300$ & $\$ 2,347,585$ & $\$ 8,386,715$ \\
\hline & $\mathrm{SA} 3 \& \mathrm{SB} 2$ & $\$ 10,694,340$ & $\$ 2,331,619$ & $\$ 8,362,721$ \\
\hline & SA3 \& SB3 & $\$ 10,757,400$ & $\$ 3,227,663$ & $\$ 7,529,737$ \\
\hline \multirow[t]{9}{*}{12 months } & SA1 \& SB1 & $\$ 21,517,140$ & $\$ 4,180,719$ & $\$ 17,336,421$ \\
\hline & SA1 \& SB2 & $\$ 21,485,160$ & $\$ 4,147,729$ & $\$ 17,337,431$ \\
\hline & SA1 \& SB3 & $\$ 21,535,200$ & $\$ 7,567,193$ & $\$ 13,968,007$ \\
\hline & SA2 \& SB1 & $\$ 21,484,500$ & $\$ 4,296,393$ & $\$ 17,188,107$ \\
\hline & $\mathrm{SA} 2 \& \mathrm{SB} 2$ & $\$ 21,458,280$ & $\$ 4,254,077$ & $\$ 17,204,203$ \\
\hline & $\mathrm{SA} 2 \& \mathrm{SB} 3$ & $\$ 21,497,100$ & $\$ 7,682,932$ & $\$ 13,814,168$ \\
\hline & SA3 \& SB1 & $\$ 21,533,760$ & $\$ 5,016,754$ & $\$ 16,517,006$ \\
\hline & $\mathrm{SA} 3 \& \mathrm{SB} 2$ & $\$ 21,495,480$ & $\$ 4,984,475$ & $\$ 16,511,005$ \\
\hline & SA3 \& SB3 & $\$ 21,558,480$ & $\$ 8,398,857$ & $\$ 13,159,623$ \\
\hline \multirow[t]{9}{*}{24 months } & SA1 \& SB1 & $\$ 43,120,320$ & $\$ 8,356,541$ & $\$ 34,763,779$ \\
\hline & SA1 \& SB2 & $\$ 43,084,860$ & $\$ 8,290,833$ & $\$ 34,794,027$ \\
\hline & SA1 \& SB3 & $\$ 43,135,020$ & $\$ 21,614,861$ & $\$ 21,520,159$ \\
\hline & $\mathrm{SA} 2 \& \mathrm{SB} 1$ & $\$ 43,089,480$ & $\$ 8,589,250$ & $\$ 34,500,230$ \\
\hline & $\mathrm{SA} 2 \& \mathrm{SB} 2$ & $\$ 43,054,140$ & $\$ 8,507,900$ & $\$ 34,546,240$ \\
\hline & $\mathrm{SA} 2 \& \mathrm{SB} 3$ & $\$ 43,099,020$ & $\$ 21,843,568$ & $\$ 21,255,452$ \\
\hline & $\mathrm{SA} 3 \& \mathrm{SB} 1$ & $\$ 43,132,980$ & $\$ 11,334,105$ & $\$ 31,798,875$ \\
\hline & $\mathrm{SA} 3 \& \mathrm{SB} 2$ & $\$ 43,099,260$ & $\$ 11,268,035$ & $\$ 31,831,225$ \\
\hline & $\mathrm{SA} 3 \& \mathrm{SB} 3$ & $\$ 43,158,840$ & $\$ 24,572,303$ & $\$ 18,586,537$ \\
\hline
\end{tabular}

\section{CONCLUSION AND FUTURE WORK}

The framework of supply chain simulation using web services was investigated to enhance the interoperability and reusability of supply chain simulation modeling and analysis. The prototype developed here shows that the major components necessary to implement the proposed framework, including simulation services, BPEL modeling and BPEL execution, can be readily developed using web services technology. In particular, the simple supply chain simulation model and the associated analysis reveals the crucial role simulation can plan in strategic decision making. Specifically, the simulation results indicate that the optimal combination of suppliers changes depending on the simulation period. Application of the proposed supply chain simulation can thus provide vital information support for decision making. 
Although the proposed framework demonstrates the viability of adopting web services for supply chain simulation, several challenges remain. The first challenge is the supply chain simulation modeling. The current supply chain model for proposed simulation framework is designed for pull production. The simulation modeling for push production should be considered in future work. The next challenge is the issue of supply chain performance. A general supply chain performance measure that can be adopted by most companies is needed. Another challenge is the development of a general PADS architecture. To generalize the proposed simulation framework, all companies should deploy their simulation model as a simulation service. The input, output, and production policy of simulation services should be generally defined for most companies. Additionally, the methodology to manage the simulation time in PADS should be considered. Finally, a methodology is needed to advertise simulation services such as UDDI. To invoke a simulation service in a heterogeneous simulation environment, the invocation method should be registered in a public registry.

\section{REFERENCES}

Banks, J., S. Buckley, S. Jain, P. Lendermann and M. Manivannan 2002. Panel session: oppertunities for simulation in supply chain management. In Proceeding of the 2002 Winter Simulation Conference, ed. E. Yücesan, C.-H. Chen, J. L. Snowdon and J. M. Charnes, 1652 - 1658. Piscataway, New Jersey: Institute of Electrical and Electronics Engineers, Inc.

Chandra, C. and N. Chilov 2001. Simulation modeling for information management in a supply chain. In Proceedings of Twelfth Annual Conference of the Production and Operations Management Society, ed., http://pomsmeetings.org/Meeting2001/2001/cd/papers/pdf/Chandra_2.pdf: Open publishing

Chandrasekaran, S., G. Silver, J. A. Miller, J. Cardoso and A. P. Sheth 2002. XML-based modeling and simulation: web service technologies and their synergy with simulation. In Proceedings of the 2002 Winter Simulation Conference, ed. E. Yücesan, C.-H. Chen, J. L. Snowdon and J. M. Charnes, 606 - 615. Piscataway, New Jersey: Institute of Electrical and Electronics Engineers, Inc.

Fujimoto, R. M. 1999. Parallel and distributed simulation. In Proceedings of the 1999 Winter Simulation Conference, ed. P. A. Farrington, H. B. Nembhard, D. T. Sturrock and G. W. Evans, 122 - 131. Piscataway, New Jersey: Institute of Electrical and Electronics Engineers, Inc.

Gan, B. P., L. Liu, S. Jain, S. J. Tumer, W. Cai and W.-J. Hsu 2000. Distributed supply chain simulation across enterprise boundaries. In Proceedings of the 2000 Winter Simulation Conference, ed. J. A. Joines, R. R. Barton, K. Kang and P. A. Fishwick, 1245-1251. Piscataway, New Jersey: Institute of Electrical and Electronics Engineers, Inc.

Kilgore, R. A. 2002. Simulation web services with .NET technology. In Proceeding of the 2002 Winter Simulation Conference, ed. E. Yücesan, C.-H. Chen, J. L. Snowdon and J. M. Charnes, 841-846. Piscataway, New Jersey: Institute of Electrical and Electronics Engineers, Inc.

Li, B. H., X. Chai, Y. Di, H. Yu, Z. Du and X. Peng 2005. Research on service oriented simulation grid. In Proceedings of the 2005 ISADS, ed., 7-14.

McLean, C. and F. Riddick 2000. Simulation in the international IMS MISSION project: the IMS MISSION architecture for distributed manufacturing simulation. In Proceedings of the 2000 Winter Simulation Conference, ed. J. A. Joines, R. R. Barton, K. Kang and P. A. Fishwick, 1539 - 1548. Piscataway, New Jersey: Institute of Electrical and Electronics Engineers, Inc.

OASIS. 2007. Web Services Business Process Execution Language V2.0.

OMG. 2006. Business process modeling notation specification.

Oracle. 2005. Oracle BPEL Process Manager.

Page, E. H., D. M. Nicol, O. Balci, R. M. Fujimoto, P. A. Fishwick, P. L’Ecuyer and R. Smith 1999. Strategic directions in simulation research. In Proceedings of the 1999 Winter Simulation Conference, ed. P. A. Farrington, H. B. Nembhard, D. T. Sturrock and G. W. Evans, 1509 - 1520. Piscataway, New Jersey: Institute of Electrical and Electronics Engineers, Inc.

Park, A. and R. M. Fujimoto 2006. Aurora: an approach to high throughput parallel simulation. In Proceedings of the 20th Workshop on Principles of Advanced and Distributed Simulation, ed., 3-10.

Swain, J. J. 2007. Simulation software survey. In OR/MS Today, Marietta, GA, USA:LionheartPublishing, Inc.

Taylor, S. J. E. and R. Sudra 2002. Modular HLA RTI services: The GRIDS approach. In Proceedings of the 6th IEEE International Workshop on Distributed Simulation and Real-Time Applications, ed.,

Taylor, S. J. E., R. Sudra, T. Janahan, G. Tan and J. Ladbrook. 2002. GRIDS-SCF: an infrastructure for distributed supply chain simulation. SIMULATION 78(5):312-320.

Terzi, S. and S. Cavalieri. 2004. Simulation in the supply chain context: a survey. Computers in Industry 53(1):3-16. 
Tsalgatidou, A. and T. Pilioura. 2002. An overview of standards and related technology in web services. Distributed and Parallel Database 12(2-3):135-162.

UN/CEFACT. 2005. UNeDocs: Stable data architecture for single window implementation.

Yücesan, E., Y.-C. Luo, C.-H. Chen and I. Lee. 2001. Distributed web-based simulation experiments for optimization. Simulation Practice and Theory 9(1):73-90.

\section{AUTHOR BIOGRAPHIES}

TAEJONG YOO is a Ph.D. candidate in the Industrial and Management Engineering Department at the Pohang University of Science and Technology, South Korea. His research interests include the technology of service oriented architecture, parallel and distributed simulation, supply chain simulation and its optimization algorithms. His email is <tju13@postech.ac.kr>.

KYUNGDOC KIM is a Master's candidate in the Department of Industrial and Management Engineering at Pohang University of Science and Technology. His research interest is simulation for supply chain integration and semantics problem on business document and process. His email address is $<$ kdoc 85 epostech.ac.kr $>$.

SUNYOUNG SONG is in a M.S. program in the Industrial and Management Engineering Department at the Pohang University of Science and Technology, South Korea. Her research area includes supply chain management and interoperability of information systems. Her e-mail address is $<$ mysong@postech.ac.kr>.

HYUNBO CHO is a professor of Department of Industrial and Management Engineering at the Pohang University of Science and Technology. He received his B.S. and M.S. degrees in Industrial Engineering from Seoul National University in 1986 and 1988, respectively, and his Ph.D. in Industrial Engineering with a specialization in Manufacturing Systems Engineering from Texas A\&M University in 1993. He was a recipient of the SME's 1997 Outstanding Young Manufacturing Engineer Award. His areas of expertise include Open Innovation, Manufacturing Management \& Strategy, and Supply Chain Integration. He is an active member of IIE and SME. He is also the director of KorBIT established in 2001 in order to promote the global interoperability of B2B applications. The KorBIT has been utilized to serve conformance and interoperability testing in the area of B2B messaging infrastructure and business object documents. He envisioned the KorBIT to directly benefit the various stakeholders - software vendors, users, standards organizations, and government - by coordinating the activities of internationally distributed testbed building and operations. His e-mail address is $\langle$ hcho@postech.ac. kr $>$.

ENVER YÜCESAN is a professor of Operations Management at the Technology and Operations Management Area of INSEAD. He holds an undergraduate degree in Industrial Engineering from Purdue and a doctorate in Operations Research from Cornell. His research interests are in the area of computer simulation experiments for stochastic systems and, more specifically, in the simulation methodology for ranking and selection as well as for optimization via simulation. He has served as a Proceedings co-editor for WSC'02. He will be serving as the Program Chair for WSC'10. His email address is $<$ Env- $^{-}$ er.YUCESANQinsead.edu>. 